\title{
Modeling double strand break susceptibility to interrogate structural variation in cancer
}

\author{
Tracy J. Ballinger ${ }^{1 *} \mathbb{D}$, Britta A. M. Bouwman², Reza Mirzazadeh², Silvano Garnerone ${ }^{2}$, Nicola Crosetto ${ }^{2 \dagger}$ and \\ Colin A. Semple ${ }^{1+}$
}

\begin{abstract}
Background: Structural variants (SVs) are known to play important roles in a variety of cancers, but their origins and functional consequences are still poorly understood. Many SVs are thought to emerge from errors in the repair processes following DNA double strand breaks (DSBs).

Results: We used experimentally quantified DSB frequencies in cell lines with matched chromatin and sequence features to derive the first quantitative genome-wide models of DSB susceptibility. These models are accurate and provide novel insights into the mutational mechanisms generating DSBs. Models trained in one cell type can be successfully applied to others, but a substantial proportion of DSBs appear to reflect cell type-specific processes. Using model predictions as a proxy for susceptibility to DSBs in tumors, many SV-enriched regions appear to be poorly explained by selectively neutral mutational bias alone. A substantial number of these regions show unexpectedly high SV breakpoint frequencies given their predicted susceptibility to mutation and are therefore credible targets of positive selection in tumors. These putatively positively selected SV hotspots are enriched for genes previously shown to be oncogenic. In contrast, several hundred regions across the genome show unexpectedly low levels of SVs, given their relatively high susceptibility to mutation. These novel coldspot regions appear to be subject to purifying selection in tumors and are enriched for active promoters and enhancers.
\end{abstract}

Conclusions: We conclude that models of DSB susceptibility offer a rigorous approach to the inference of SVs putatively subject to selection in tumors.

Keywords: Double strand break, Cancer, Structural variation, Chromatin, Modeling

\section{Background}

Structural variation (SV) in tumor genomes is known to play important roles in disease progression and may be critical in driving the development of certain cancer types [1-3]. However, challenges remain not only in ascertaining accurate SV calls, as evidenced by the compendium of SV calling algorithms used in many projects [4-6], but also in predicting their functional impact. Some SVs have apparently direct consequences; for example, amplification of oncogenes leading to overexpression, deletion of tumor suppressors leading to

\footnotetext{
* Correspondence: Tracy.Ballinger@igmm.ed.ac.uk

${ }^{\dagger}$ Nicola Crosetto and Colin A. Semple contributed equally to this work. ${ }^{1}$ MRC Human Genetics Unit, MRC Institute of Genetics and Molecular Medicine, University of Edinburgh, Crewe Road, Edinburgh EH4 2XU, UK Full list of author information is available at the end of the article
}

dysfunction, and translocations generating oncogenic fusion proteins [4]. Reportedly indirect consequences of SVs include changes in enhancer targeting, affecting the expression of nearby genes, or "enhancer hijacking" [7]. However, it remains challenging to distinguish the influences of evolutionary selection versus primary mutation rate in generating the SVs concerned.

A recent study of whole genome sequencing (WGS) data from breast tumors identified SV hotspots and putative driver SVs, but could not discern the relative contributions of mutational bias and selection underlying these hotspots [8]. Resolving the influences of mutational bias versus selective forces has become critical given that both single nucleotide variant (SNV) and SV mutation rates vary widely across the genome, in parallel

(c) The Author(s). 2019 Open Access This article is distributed under the terms of the Creative Commons Attribution 4.0 International License (http://creativecommons.org/licenses/by/4.0/), which permits unrestricted use, distribution, and 
with replication timing and chromatin structure $[9,10]$. In analyses of tumor SNVs, variants are routinely prioritized based on algorithms including corrections for estimates of SNV mutation rate variation [11], but analogous methods are not yet applied to SVs.

Variable rates of SVs observed across the genome are likely to be affected by differences in the efficiency of repair of DNA double strand breaks (DSBs). DSBs can be repaired by homologous recombination (HR) at the G2 and $\mathrm{S}$ stages of the cell cycle and, more commonly, by canonical non-homologous end joining (c-NHEJ) which operates throughout the cell cycle [12]. The c-NHEJ process is error prone and has been shown to create structural variants initiating carcinogenesis [13]. A third repair process, alternative NHEJ (alt-NHEJ) uses microhomology to mediate repairs when the c-NHEJ pathway is unavailable, and repair by alt-NHEJ appears to increase the rate of deletions, insertions, and translocations further [14]. The efficiency of these repair processes is often dependent upon the chromatin features and nuclear organization present where the damage occurs. For example, the histone modification H3K36me3, associated with active transcription, recruits the HR pathway, while H4K20me1, a mark of highly transcribed genes, recruits components of the NHEJ pathway [15]. The associations between DSB repair and the underlying chromatin landscape may, therefore, explain the observed correlations between tumor SV rates and chromatin structure [9].

Previous studies have also shown DSB formation to be influenced by underlying chromatin structures and genomic sequences. It has long been known that certain cytogenetically mapped loci, termed "fragile sites" undergo recurrent DSBs in cells under replicative stress and in cancer [16]. More recent high-throughput sequencing (HTS)-based approaches have been developed to profile DSB rates more precisely within in vitro populations of cells [17-25]. Three of these methods, BLESS [18], DSBCapture [22], and BLISS [25], are closely related and have been used to generate high-resolution maps of endogenous DSBs occurring in human cell lines, resulting in continuous data reflecting the propensities for DSBs across all chromosomes. These studies have suggested that DSBs may preferentially occur within nucleosome-depleted regions, are correlated with active promoter and enhancer histone modifications, and may associate with G-quadruplex sites [22, 26]. Certain studies have also suggested DSBs to be depleted in some transposon classes and enriched in some simple repeat classes and to be unusually frequent in long, late-replicating genes [18, 24]. Overall, previous studies have found correlations and enrichments between DSBs and various inter-correlated chromatin and genomic features, making it difficult to accurately assess the contribution of any particular feature to DSB susceptibility. Understanding such contributions can be valuable for understanding the underlying mutational and repair mechanisms. In addition, a fuller understanding of the relative contributions of many features to DSB formation can allow reliable predictions of the expected DSB frequency in a given genomic region.

Random forests have been used to model a variety of biological phenomena because they perform well in the presence of inter-correlated input variables showing non-linear relationships. For example, they have been used to predict nuclear compartments [27], cancer SNV mutational landscapes [28], and enhancer-promoter interactions [29]. In this study, we construct random forest regression models to generate quantitative measures of the relative importance of a variety of matched chromatin and other features to DSB susceptibility. We use multiple, high-resolution DSB profiling datasets to compare modeling accuracy across several platforms and cell types. The cell types selected have also been extensively profiled for a variety of chromatin features by the ENCODE Project [30] and others, allowing well-matched models to be constructed for all datasets. We demonstrate that these models provide accurate estimates for the expected rate of DSBs in a given region and can be cross applied between DSB datasets. In addition, the models can be used to explore tumor SV breakpoint data, to nominate novel regions putatively subject to selection in cancer.

\section{Results}

We uniformly processed four DSB datasets from three related platforms (DSBCapture and BLISS are both based upon modifications to the BLESS protocol) and covering three different cell types, collating matched chromatin data for each. These datasets include two novel DSB mapping datasets derived from the K562 erythroleukemia and MCF7 breast cancer cell lines using the recently developed BLISS method [25] (see the "Methods" section) and two previously published DSB mapping datasets derived from the NHEK keratinocyte cell line using BLESS and DSBCapture [22] protocols. DSB frequency is defined in each dataset as the number of unique reads mapping to a given $50 \mathrm{~kb}$ region, since each read in a DSBCapture, BLESS, or BLISS experiment represents an exposed DNA DSB end. Replicate experiments within each dataset were strongly and significantly correlated (Pearson's $r=0.905$ to $0.992, p<2.2 \mathrm{e}$ $-16)$ and were combined to reduce noise, although random forest models generated from any single one of the replicates yielded very similar results (see the "Methods" section). Comparisons among DSB profiling datasets showed moderate correlations in genome-wide DSB frequency between the three cell types as expected $(r=$ 
0.351 to $0.635, p<2.2 \mathrm{e}-16$ ), shown in Additional file 1: Figure S1. All three cell types correspond to well-characterized ENCODE cell lines, providing numerous matched chromatin and genomic features exhibiting a range of correlations to DSB (Fig. 1), and are also inter-correlated themselves (Additional file 1: Figure S2).

\section{Accurate models of genome-wide DSB frequency across cell types}

We modeled DSB frequency at $50 \mathrm{~kb}$ resolution, using the same ten matched genomic features from each cell type to construct random forest models (see the "Methods" section): open chromatin assayed by DNase-seq, POL2B binding, CTCF binding and five histone modifications assayed by ChIP-seq, replication timing assayed by Repli-seq, and RNA-seq. We also included G-quadruplex forming regions as an additional feature, since these DNA secondary structures are associated with genomic instability [31]. We found strong and significant correlations between predicted and observed DSB frequency for all four datasets, with Pearson's coefficients ranging from 0.83 to
0.92 (Fig. 2). We also generated a model for the NHEK DSBCapture dataset using an extended set of 21 features, including additional histone modifications, histone variants, and nuclear compartmentalization from $\mathrm{Hi}-\mathrm{C}$ data [32]. This extended model resulted in better predictive results for a small fraction of the genome (Additional file 1: Figure S4, Box B), and a modestly increased genome-wide Pearson's coefficient between predicted and observed values (11 feature model $r=0.918,21$ feature model $r=$ 0.922). We conclude that models constructed using the 11 selected genomic features (Fig. 2) provide high predictive accuracy across cell types, with additional features likely to provide only marginal gains.

Variable importance metrics for these models reveal consistent trends in the most influential features in DSB frequency prediction (Fig. 2e-h). Replication timing is the most important feature across all three models with early replication associated with high DSB regions and late replication with low DSB (Fig. 3c), in agreement with previous studies [33]. In addition, the histone modifications H3K36me3 and H3K9me3 (demarcating active

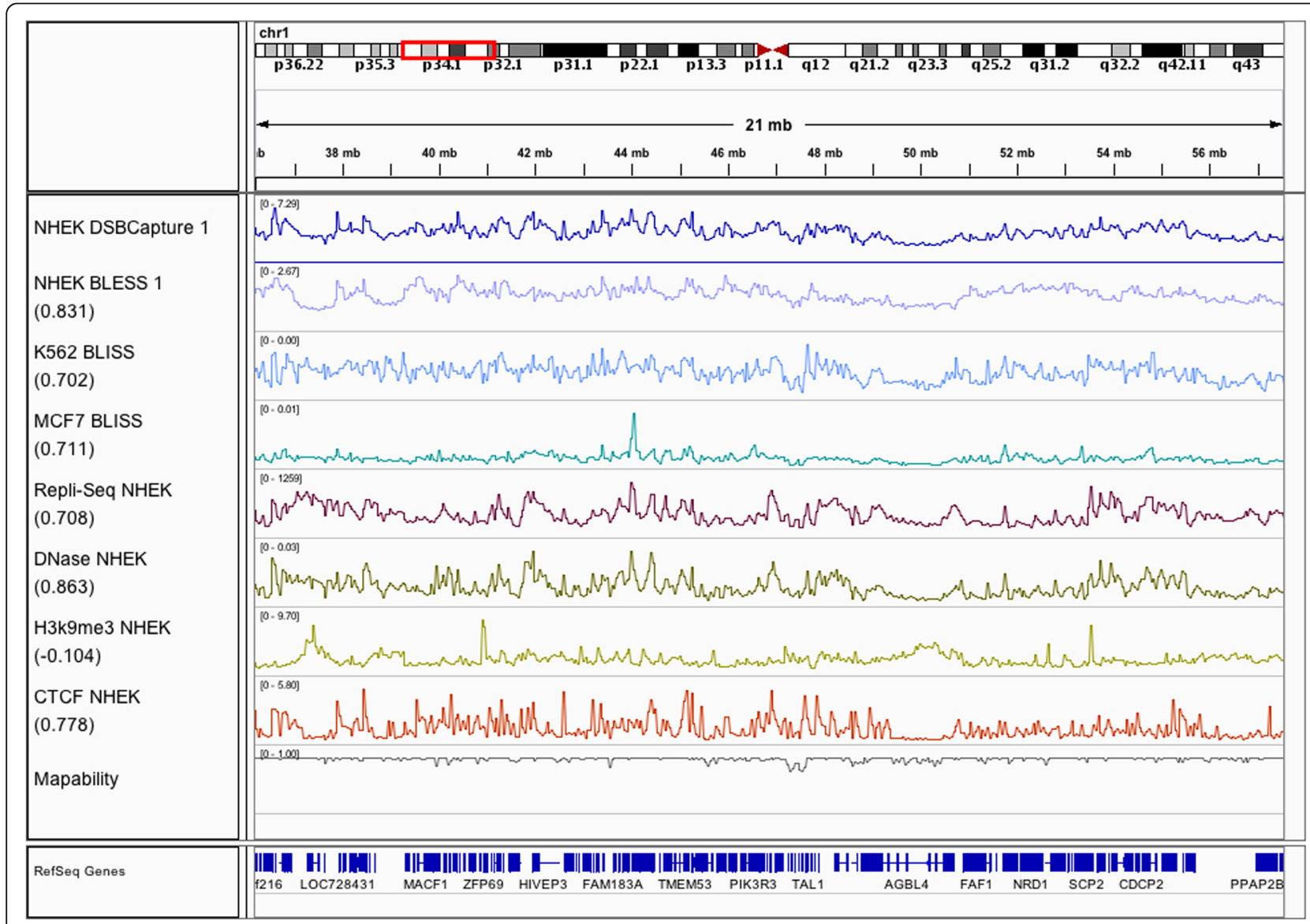

Fig. 1 DSB frequency and genomic features display similar patterns. The tracks show DSBCapture profiles in NHEK cells, BLESS profiles in NHEK cells, BLISS in K562 cells, and BLISS in MCF7 cells. All tracks are at $50 \mathrm{~kb}$ resolution over a representative region of chromosome 1, with a variety of chromatin and sequence features to illustrate the similarities between them. Numbers in parenthesis are the Spearman's rho between the associated track and the NHEK DSBCapture 1 dataset 


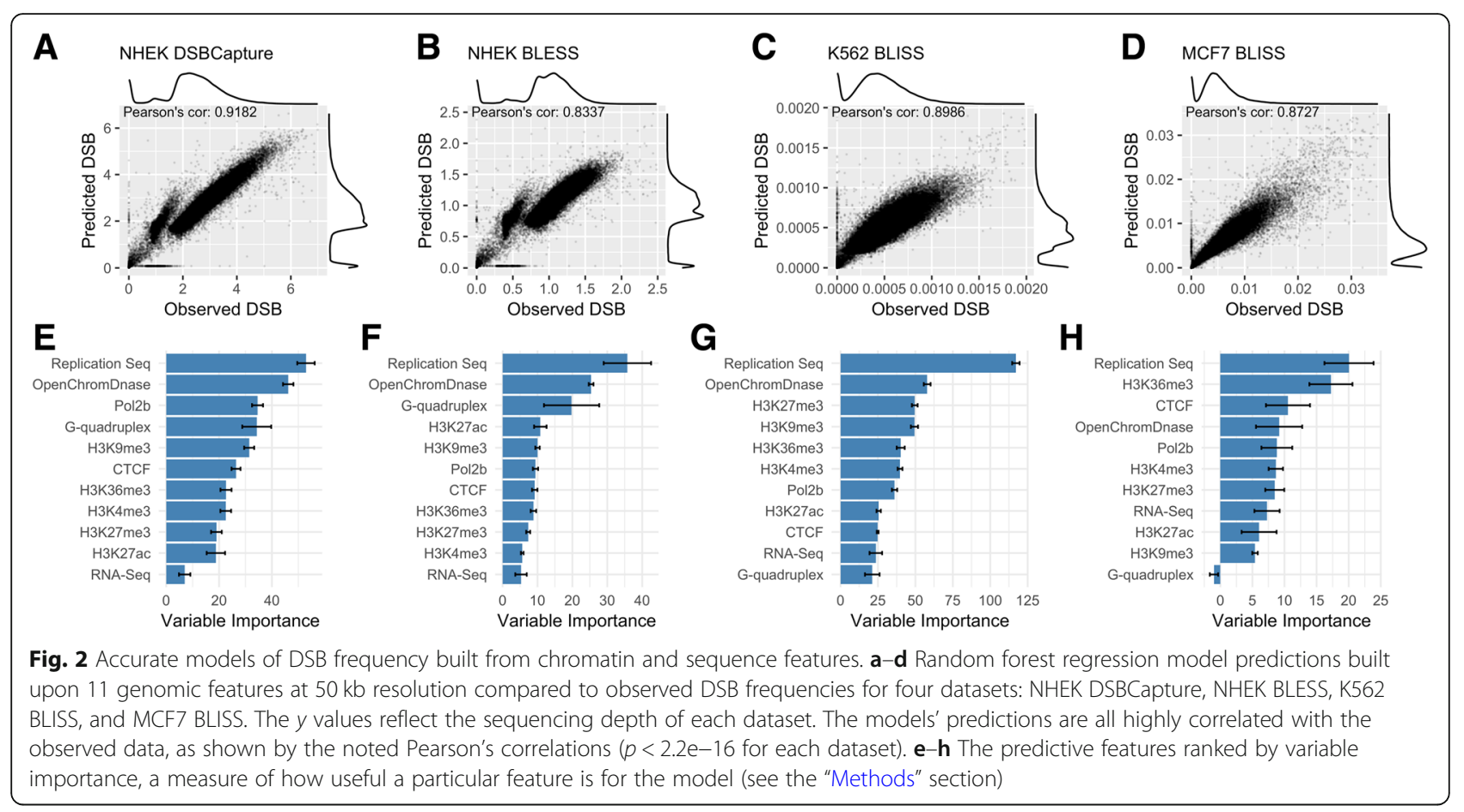

genes and gene-poor heterochromatin respectively) emerge as informative features, with H3K36m3 enriched in high DSB regions and H3K9me3 in low DSB regions (Fig. 3c). This is consistent with observations that structural variants disproportionately accumulate within the early replicating, relatively gene-rich regions of the genome in cancer, and are relatively depleted in late replicating heterochromatin $[9,10]$. DNase-seq open chromatin ranks second in three datasets and fourth in the MCF7 model and is also the most important feature for predicting DSB peaks in the study of Mourad et al. [34] in which they do not include replication timing. The influence of G-quadruplex forming regions is notably variable, ranking as a relatively important feature in the NHEK datasets, but having little and no predictive value in the $\mathrm{K} 562$ and MCF7 datasets. RNA-seq is not a strong predictor of DSB susceptibility although DNase-seq peaks are often found at the promoter regions of active genes. This suggests that open chromatin at transcriptionally active genes and associated regulatory elements (reflected in DNase-seq, H3K4me3, and POL2B binding), rather than transcription per se, is the dominant influence on DSB frequency. CTCF binding also appears to be an informative variable, genome-wide in all models, though it binds at sites constituting a very small fraction of the genome. Given the critical roles of CTCF in chromatin architecture and regulation [32], there has been intense interest in the causes and effects of structural variants disrupting CTCF binding sites $[35,36]$.
Influential features underlying DSB frequency differ between genomic loci and cell types

Beyond the general, genome-wide trends described above, we see differences in the behavior of certain classes of loci. These are evident as regions departing from the linear relationship between observed and predicted DSB frequency seen for the majority of the genome (Fig. 3a; Additional file 1: Figure S4). Deeper exploration of the relationships between underlying genomic features and DSB frequency reveals diagnostic features for these discrepant classes. One class of loci (Fig. 3, Box A) shows unusually low values for both predicted and observed DSB frequencies and is enriched for H3K9me3 marked heterochromatin and low sequence mappability (Fig. 3b). These regions are likely to correspond to repeat-rich regions near centromeres and on the short arms of acrocentric chromosomes, which are problematic for read mapping algorithms [37]. Another class of H3K9me3 heterochromatin enriched loci shows higher DSB predictions than observed, in spite of high mappability values (Fig. 3, Box B). This class of regions is absent in DSB datasets generated by the BLISS protocol (Fig. 2), so these aberrant predictions may reflect technical and methodological differences between datasets. In any case, it is clear that model predictions may reasonably be expected to be less accurate in heterochromatic regions.

The similarities in relative variable importance across datasets (Fig. 2) suggest that many features have a similar influence on DSB frequency in each of the three cell 


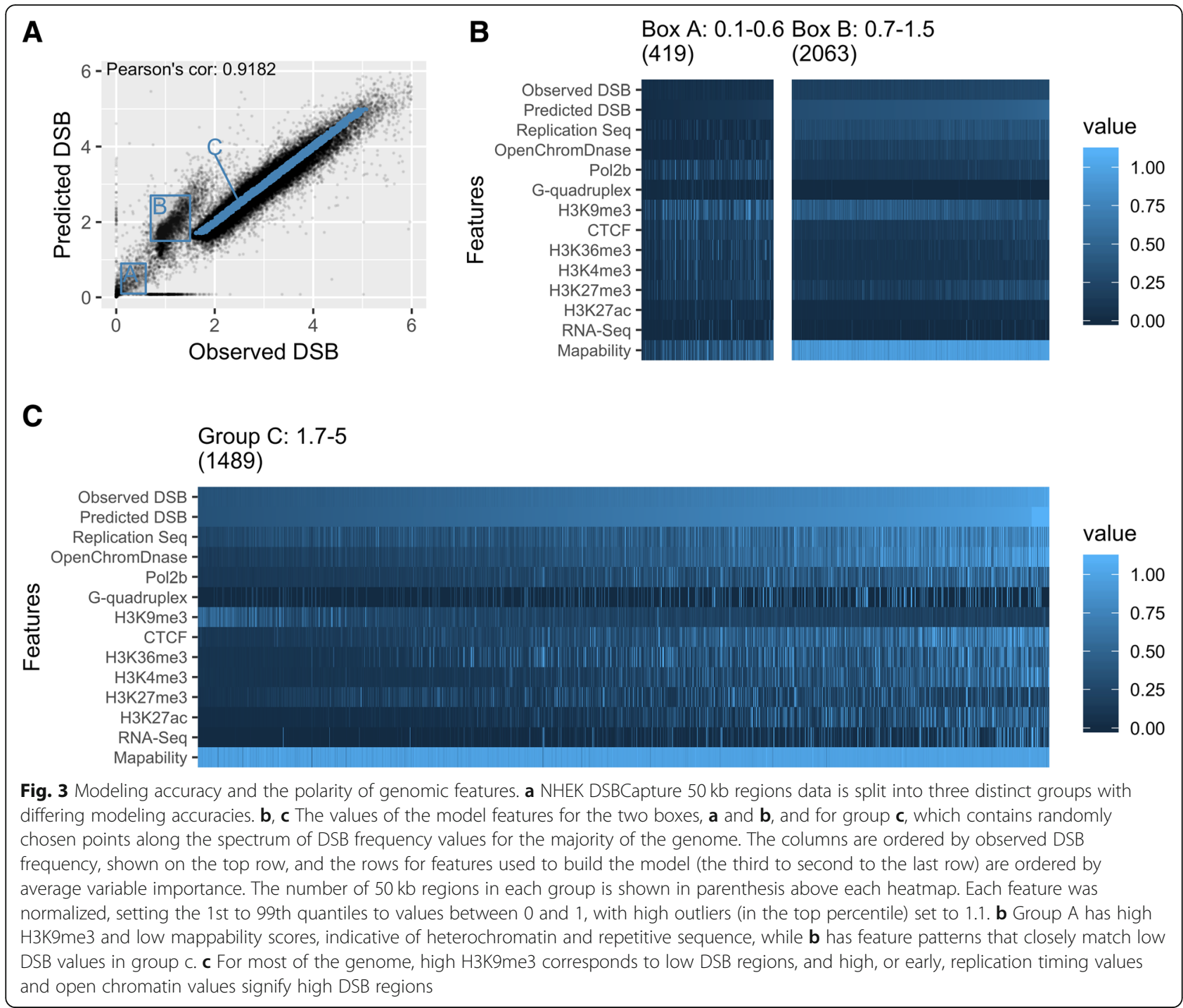

types. Thus, a model trained in one cell type might generalize well to another cell type and allow us to generate predictive DSB frequency profiles for model cell lines currently lacking high-resolution DSB data. We cross-applied models and found models trained in one cell type often performed well in another (Fig. 4). For example, a model trained in NHEK cells could be used to predict DSB frequencies in K562 cells (inputting K562 genomic features) with high accuracy (Pearson's $r=0.85$ correlation; Fig. 4). This offers a substantial improvement over the base correlation $(r=0.63)$ between NHEK and $\mathrm{K} 562$ observed DSB profiles. We measured the correlation of observed and predicted DSB frequencies across all nine model and feature combinations and always found correlations ( $r=0.58$ to 0.85 ) that improved on the base correlations ( $r=0.38$ to 0.63 ) seen between the observed DSB datasets (Fig. 4). These improvements echo the similarities in variable importance between cell types (Fig. 2). The moderate correlations between DSB across cell types demonstrate that a substantial proportion of DSB susceptibility across the genome is cell type specific, which is consistent with the established cell type-specific properties of many SV breakpoint regions in tumors, such as common fragile sites [38]. Furthermore, the larger performance gap in models for cell lines with altered variable rankings indicates that DSB mechanisms may differ across cell types and may not be completely captured via epigenomic features.

\section{Tumor SV breakpoints possess variable susceptibility to DSBs}

Keratinocytes are considered to be the cell type of origin for mucosal and cutaneous carcinomas, particularly squamous cell carcinomas [39], and NHEK cells are often used in the literature as a model for these cancers. Similarly, MCF7 cells and K562 cells have been used 


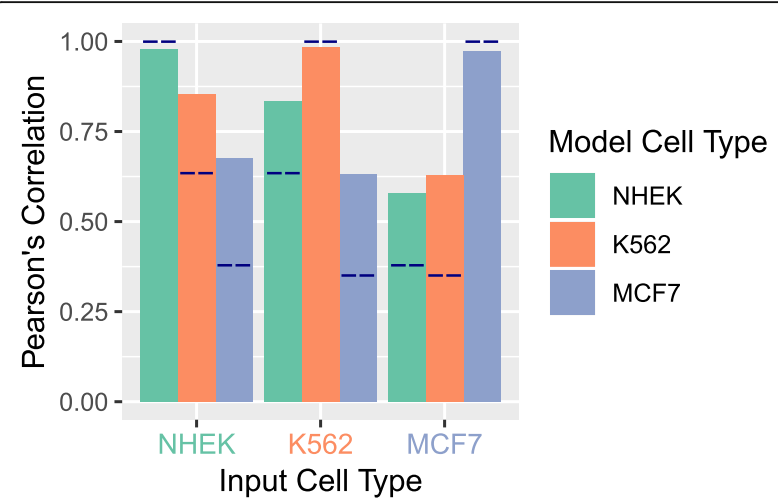

Fig. 4 DSB models improve predictions for non-model cell types. Models trained using a dataset from one cell type were used to generate predictions for a different cell type, given the matched features. The dark blue lines mark the Pearson's correlation between the two cell types. The cell type used to train the model is indicated by the color of the bar, and the cell type on which the model is being applied is shown on the $x$-axis. In all cases, the random forest model greatly improves the predictions from a naive inference, with a 1.3-1.8 fold improvement in correlation

extensively as models for breast and blood cancers respectively. This motivated us to ask how the DSB models for these three cell types relate to the patterns of SV breakpoints observed in squamous cell carcinomas, blood cancers, and breast tumors.

A number of large structural variant (SV) collections have been established for a variety of tumor types, and each possesses advantages and shortcomings. The International Cancer Genome Consortium (ICGC) provides high-resolution SV calls based upon whole genome sequencing (WGS) for 2146 patients across 17 cohorts [40], but sample cellularities, sequencing depths, and SV calling methods vary across cancer cohorts and are expected to affect results (Additional file 1: Figure S6). The Cancer Genome Atlas (TCGA) produced consistently processed copy number variant (CNV) calls from SNP chip data for 23,084 patients across 33 cohorts (Additional file 1: Figure S7). However, breakpoint resolution is much lower than calls based upon WGS, and copy neutral SVs such as inversions and translocations are absent. We analyzed ICGC and TCGA data as pancancer datasets, combining all cancer types together, but also as three cancer type subgroups. TCGA subgroups comprised a squamous cell carcinoma subgroup, a blood cancers subgroup including two blood cancers, and breast cancer as a separate group (see the "Methods" section). Similar ICGC subgroups were formed (from cohorts independent of TCGA), but with the squamous cell carcinoma subgroup replaced with a carcinoma subgroup, which includes seven carcinoma cancer studies excluding breast cancer (see the "Methods" section).
Analogously to the DSB datasets, we determined the number of tumor SV breakpoints per $50 \mathrm{~kb}$ region for each of the ICGC and TCGA SV datasets (see the "Methods" section) and compared these to the DSB predictions from our models. In ICGC data overall we saw low correlations between the number of SV breakpoints and DSB predictions (Additional file 1: Figure S8 and S9). Restricting our analysis to ICGC enriched SV breakpoint regions, or ESBs for the purpose of this manuscript (50 kb regions with SV breakpoint counts in the top 5\% genome-wide, see the "Methods" section), increased the agreement with DSB model predictions. Significant increases in NHEK and MCF7 model predictions were seen for pancancer, carcinoma, blood, and breast tumor ESBs and in K562 model predictions for all cancer subsets except blood ESBs (Fig. 5). The significant increase in DSB model predictions seen for carcinoma ESBs indicates that DSB susceptibility (captured in the models) may shape the SV landscape of these cancer types. We also see a significant increase in DSB predictions for TCGA blood cancer ESBs, but not for any other subgroups in TCGA data (Additional file 1: Figure S10). However, as mentioned, TCGA data is of low resolution and not suitable for accurate breakpoint detection.

Certain classes of relatively simple SVs (deletions, duplications, inversions, translocations) are often the product of one or two DSBs, while more complex intrachromosomal rearrangements can be difficult to classify accurately, and may have origins in poorly understood phenomena such as chromothripsis [41]. Indeed, even for simple SVs, there may be some ambiguity, with an unknown fraction arising by mechanisms that may not involve a DSB. For example, insertions can arise from transposon activity, and duplications from replication slippage [42]. However, even if many SV breakpoints do not arise from DSBs, we might reasonably expect to see shifts to higher median DSB model prediction values for many simple SV classes. We determined ESBs as above for ICGC-annotated SV classes across all ICGC tumor types to examine their DSB frequency predictions, compared to non-ESBs, 50 $\mathrm{kb}$ regions that do not attain SV breakpoint counts in the top 5\% with at least one tumor SV breakpoint detected. Overall, the models show significant elevations for ESBs covering all SV classes except insertions (Fig. 5). Insertions may be less influenced by DSB susceptibility because they may occur via transposable element activity rather than through DNA damage and repair pathways. Crosetto et al. [18] find an enrichment of satellite repetitive elements in regions enriched for DSB in cells exposed to aphidicolin. However, regions that undergo DSB under replicative stress, as induced by aphidicolin, may differ from DSB regions under normal cell growth conditions. 


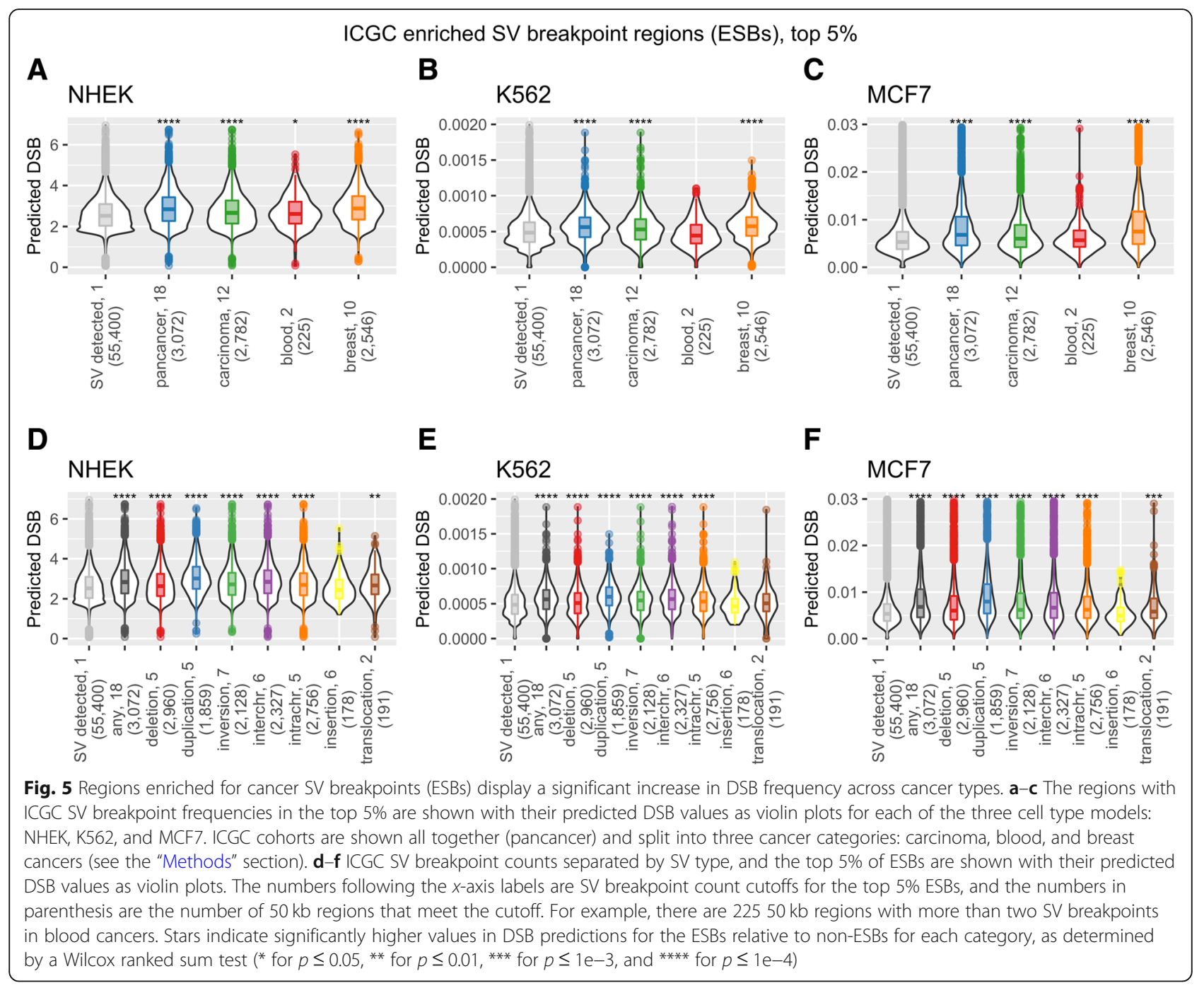

\section{Interrogating tumor SV data at common fragile sites with} DSB models

The predicted DSB frequencies from our models and ICGC tumor SV breakpoint frequencies differ in their scaling and distributions and are not directly comparable. However, it is of interest to identify outlier regions, where model predictions and observed tumor SV breakpoint rates diverge most, since these regions may include loci under selection in tumors. We developed a novel metric, the $d$-score, to measure this divergence between expectations given a DSB model and observed SV breakpoint rates in tumors. In brief, this metric relies on fitting known distributions to the observed SV breakpoint dataset and to the predicted DSB dataset. Based upon the known distributions we then transform the observed SV counts and predicted DSB values to $p$ values, reflecting the probability that each value is drawn from the fitted distribution (see the "Methods" section). For each $50 \mathrm{~kb}$ region in the genome, the difference between the SV breakpoint $\log p$ value and the predicted DSB $\log p$ value is the $d$-score. Regions with unexpectedly high $d$-scores contain more SV breakpoints than expected, given our model, whereas regions with unusually low $d$-scores contain fewer SV breakpoints than expected.

Common fragile sites (CFSs) have long been studied for their unusual properties of generating SVs, both in normal cells and in cancer [38]. These regions undergo frequent DSBs in tumors and have been well studied in terms of their genomic context, relationship to replication timing and origins, and correlations with particular chromatin states [43]. They tend to occur within large genes, in G-negative chromosomal bands with high DNA flexibility, are unusually late replicating [44], and it is thought that their instability derives from transcription-associated replication stress [38]. CFSs only exist in modest numbers and are defined at low resolution (by cytogenetic bands or gene loci); they, therefore, provide an interesting, though challenging, test set of regions to examine $d$-score performance. 
We examined predicted (NHEK model) DSB frequencies at $29450 \mathrm{~kb}$ regions coinciding with annotated CFS gene loci across the genome, in comparison to regions associated with all annotated genes, and regions associated with putative cancer driver genes (Fig. 6c). Although significant shifts to higher frequencies are seen for the driver gene sets for predicted DSB frequencies, the CFSs do not show a similar increase, most likely because the model predicts DSB in early replicating regions, and CFS tend to be late-replicating. Thus, the dominant features influencing DSB susceptibility genome-wide do not appear to drive the elevated DSB rates at CFSs, consistent with CFS instability involving replicative stress [38]. However, CFS $d$-scores show a significant shift above the distribution for all genes and above the driver gene sets as well (Fig. 6d). This result is replicated in the MCF7 BLISS model examined in conjunction with ICGC breast cancer SV breakpoints (Additional file 1: Figure S11). We conclude that the $d$-score, a measure of relative DSB enrichment, offers a robust metric for the classification of regions showing unusual SV breakpoint rates in tumors.

\section{Identification of hot and cold spots for structural variant breakpoints in tumors}

We have developed a classification of regions of interest within ICGC tumor cohorts based upon the $d$-score metric. We call regions with significantly more SV breakpoints than expected, or SV hotspots, cancHpredL (cancer high, predicted low), and regions with fewer SV breakpoints than expected, or SV coldspots, cancLpredH (cancer low, predicted high) (see the "Methods" section).

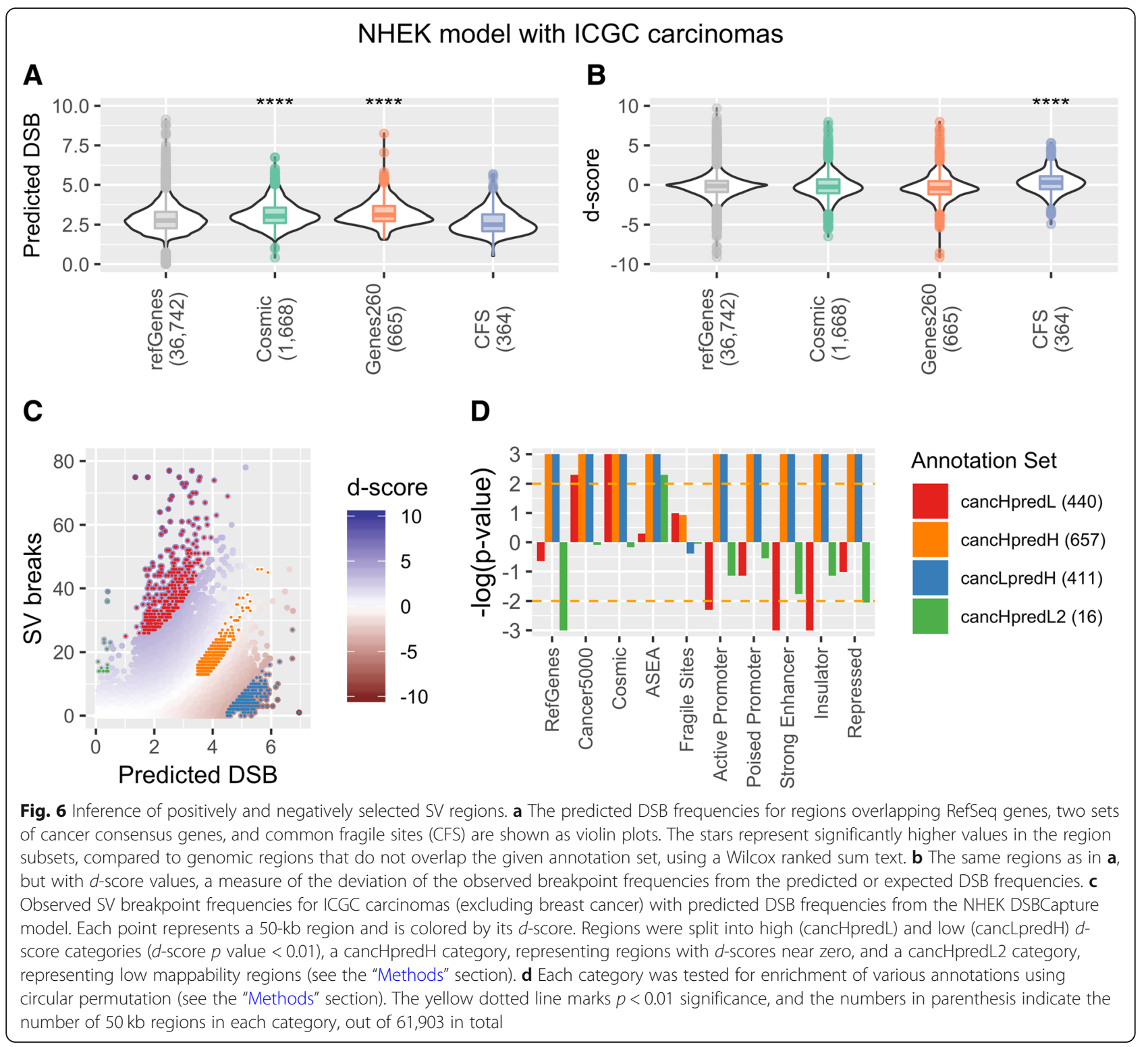


Figure 6 depicts these classes of regions in $d$-score plots of ICGC SV breakpoint data. Many previous studies have predicted oncogenic SV hotspots simply as regions repeatedly rearranged in cancers. Here, we refine such predictions by assessing these raw SV breakpoint frequencies relative to the predicted susceptibility of each region to breakage. It is not possible to predict coldspot regions without a model of expected DSB frequency, and to our knowledge, SV breakpoint coldspots have not been studied before.

We also define a class of regions possessing both high predicted DSB values and high SV breakpoint frequencies (cancHpredH), corresponding to regions showing unusually high SV frequencies on the background of high susceptibility to DSBs. Finally, we define a fourth class of regions that have predicted DSB rates close to zero but high SV breakpoint frequencies (cancHpredL2). In principle, these regions are a class of SV hotspots but, as shown in Fig. 3b, they are likely to be repetitive, heterochromatic, and enriched for artifacts (false positives and negatives in SV breakpoint) due to their association with low mappability.

We examined a range of functional annotation enrichments in the four classes of regions using circular permutation to assess significance (see the "Methods" section; Fig. 6). The annotations included two putative cancer gene sets, 260 genes from the Cancer5000 dataset [45] and 561 genes from the COSMIC collection [46]). We also included a set of 15,415 super enhancers [47], common fragile sites, and chromatin states from ENCODE chromHMM analysis [48]. Notably, the majority of genes in both cancer sets are predicted to be oncogenic based on unexpectedly high and functionally significant SNV (rather than SV) loads and are not necessarily expected to occupy regions with higher levels of SV breakpoints. In fact, both gene sets demonstrate significant enrichments in the cancHpredL class of hotspot regions (Fig. 6d), although RefSeq genes do not, suggesting that these genes may also frequently be altered in cancer through SV. The cancHpredL regions are also significantly depleted in active chromatin regions, such as promoters, enhancers, and insulator regions, most likely because these types of regions do not have low predicted DSB. The high susceptibility cancHpredH regions occupy gene-rich areas of the genome (enriched for known RefSeq genes) including both cancer genes sets, and for active promoters, strong enhancers, and insulators. This is consistent with reports that CTCF bound insulator elements suffer recurrent mutations in tumors. Likewise, the cancLpredH class of coldspot regions occupies gene-rich neighborhoods, active promoters, and strong enhancers (Fig. 6), suggesting some genes and distal regulatory regions may have experienced purifying selection in tumors.
Given the discrepancies mentioned above between ICGC and TCGA experimental platforms, data analysis, and sample cohorts, we do not expect strong agreement between ICGC and TCGA derived SV datasets. Indeed, the correlation between them is low (Spearman's rho of 0.099, $p<2.2 \mathrm{e}-16$ ), and the pancancer ESBs from either set do not significantly overlap $(p<0.99$, see the "Methods" section ). However, the cancLpredH class is again enriched in active promoter and strong enhancer regions, in accordance with the results based upon ICGC SV data (Additional file 1: Figure S12).

We again wanted to test the utility of DSB random forest models applied to different cell types by testing the accuracy of predictions made by a model trained in one cell type given features for a different cell type, as in Fig. 4. Instead of looking at the correlation between the observed and predicted DSB scores across the genome, we examined the overlap between cancHpredL, cancHpredH, and cancLpredH $50 \mathrm{~kb}$ regions for the MCF7 model versus the NHEK model, using the MCF7 model as the truth set. Subsets of $50 \mathrm{~kb}$ regions for each model were derived from MCF7 features and ICGC breast cancer SV breakpoints; only the training data for the models differ. We found a significant overlap between all three categories of $d$-score subsets, with 595/662 cancHpredL, 255/785 cancHpredH, and 253/594 cancLpredH regions detected via the NHEK model $(p<2.2 \mathrm{e}-16)$, demonstrating that a given model can be used to detect regions of interest in various cell types.

\section{Functional annotation of regions of interest}

We closely examined the ten $50 \mathrm{~kb}$ regions with the highest (cancHpredL) $d$-scores to uncover genes that might be reclassified as oncogenic due to a higher than expected SV breakpoint frequency in cancer. Likewise, we investigated the ten regions with the lowest $d$-scores (cancLpredH), which we predict to be under purifying selection, for signals of potential functionality. For this analysis, we used the NHEK model predictions paired with ICGC carcinoma SV breakpoints.

Nine out of ten regions with the highest $d$-scores overlap a gene, and four overlap COSMIC genes. CHEK2 and $C D K N 2 A$ are known tumor suppressors, and TMPRSS2 and ERG are frequently involved in translocation events forming fusion oncogenes in certain cancers. For example, it fuses with TMPRSS2 in most prostate cancers, with EWS in Ewing's sarcoma, and with FUS in AML. Two adjacent $50 \mathrm{~kb}$ regions on chr17q12 overlap GRB7 and IKZF3. GRB7 encodes a protein that interacts with epidermal growth factor receptor (EGFR), a well-known proto-oncogene, and IKZF3 is a zinc finger protein and transcription factor involved in B lymphocyte regulation and differentiation as well as chromatin remodeling. This region also corresponds to a known 
fragile site FRA17A [49]. Of the ten regions with the lowest $d$-scores, seven overlap a known gene and two known oncogenes. The oncogene, $C D C 27$, or cell division cycle 27, encodes a component of the $A P C$ and has been shown to interact with other mitotic checkpoint proteins. It is highly conserved and may be necessary for cell survival. There is also a non-coding RNA found on chr2 in the centromeric region, LOC654342, which overlaps an H3K27ac peak, and may be acting as a regulatory element.

\section{Discussion}

Recent in vitro studies of DSB frequency in cell lines have suggested that a variety of underlying genomic features are associated with DSB susceptibility. We have shown that accurate models of genome-wide DSB frequency can be built from a modest number of such features, with replication timing, open chromatin, and marks of active promoter or enhancer regions associated with increased DSBs. Although active regulatory regions often harbor actively transcribed genes, it appears that chromatin accessibility at these sites rather than transcription itself determines DSB propensity. The variable importance metrics also show certain features to be more influential in particular cell types, with CTCF and H3K36me3 having more predictive power in MCF7 than in NHEK or K562. Not only are DSB patterns cell type specific, but the factors influencing those patterns also depend on cell type, suggesting different mutational mechanisms at play. As a matter of course, our models' accuracies decline when applied to cell lines other than the training set, but they still generate reasonable DSB frequency predictions, with correlations between 0.57 and 0.83 to the observed data, which are large improvements over a simple inference. Since chromatin features influence mutation patterns and are cell type-specific, it will be important to use mutational propensity profiles for matched cell types in future cancer studies. In general, caution should be exercised in extrapolating from the currently small number of models to more poorly characterized cell types lacking experimentally determined DSB data.

Our models of genome-wide DSB susceptibility predict DSB frequencies for all $50 \mathrm{~kb}$ loci and reflect the established correlations between replication timing and DSB frequency [50] as well as tumor SV rates $[9,10]$. A recent complementary study has shown that 84,946 high confidence peaks of NHEK DSBCapture signal [22], marking small (median: $391 \mathrm{bp}$ ) sites of unusually high DSB susceptibility, can be accurately classified from control sites using underlying genomic features [34]. Consistent with our results, this binary classifier suggested prominent roles for DNase accessible regulatory sites and CTCF binding and recapitulated many of the patterns reported by Lensing et al. (2016). However, the model of Mourad et al. (2018) omitted replication timing and does not provide quantitative predictions of DSB susceptibility across the genome.

We used our genome-wide models of DSB susceptibility to interrogate the largest tumor SV breakpoint collections and found surprising levels of agreement, such that SV breakpoint enriched regions often show shifts to higher predicted DSB susceptibility. In spite of variable sample sizes, the classes of simple SV likely to arise by one or two DSBs (deletions, duplications, inversions, translocations) showed significant increases in predicted DSB susceptibility. The NHEK model best predicted the patterns of DSB susceptibility in tumors, showing genome-wide elevations of predicted DSBs for all of these SV classes relative to control regions. Thus, the chromatin-mediated DSB susceptibility captured in the model may shape the landscape of SV recurrence in these classes.

There are many reasons why one might expect a much poorer agreement between the predictions of in vitro DSB frequency models and the patterns of SV breakpoints observed in tumor sequencing studies. The available collections of SV breakpoints in tumors are far from perfect, and even the best ICGC data suffer large variations in sample size, sample heterogeneity, sequencing depths, and SV calling methods across tumor cohorts. In addition, fundamental aspects of tumor biology (cellular heterogeneity, disrupted repair pathways, chromatin alterations etc.) are expected to place distinct limits on the agreement we can see with the DSB patterns seen in cell lines. Evidence is also emerging that there are important properties of the mutational landscape in tumors that are unlikely to be captured by in vitro model systems. For example, a recent study of intra-tumor diversification in colorectal cancer suggests that most mutations occur during the final clonal expansion of these tumors, resulting from mutational processes that are absent from normal colorectal cells [51]. Enhanced rates of DSB formation have also been observed in vitro at cryptic replication origins activated by oncogene-induced replication stress, though these cryptic sites seem to explain only a minority of SV breakpoints $(<8 \%)$ across a variety of TCGA tumor types [52]. Given the many known and possible differences between in vitro DSB model predictions and observed tumor SV breakpoints, it is remarkable that significant agreement is found on any level.

There is great interest in "hotspot" genomic regions harboring recurrent SVs in tumors, on the basis that such regions may be under positive selection, conferring a proliferative or survival advantage to tumor cells. However, a rigorous inference of selection requires a proxy for the expected rate of recurrence within such 
regions. Using model predictions as this proxy we have produced refined hotspot predictions, reflecting SV breakpoint frequencies relative to the predicted susceptibility of each region. Since our predictions of DSB susceptibility are genome-wide it was also possible to predict coldspot regions, regions possessing unexpectedly low SV breakpoint rates given model predictions, and putatively subject to negative or purifying selection in tumors. If selection in tumors is prominent in driving SV breakpoint frequencies away from DSB model predictions, we might expect hotspot and coldspot regions to show unusual functional enrichments. Multiple caveats apply to the annotations examined but analysis using the NHEK model shows that ICGC carcinoma hotspots are enriched for putative oncogenes. Coldspots occupy gene-rich neighborhoods but and are also enriched in active promoters and strong enhancers, and insulators, indicating regulatory regions that may have experienced purifying selection in tumors. Methods for the detection of DSBs in human cells, as well as the causes and consequences of these mutations, remain active areas of fundamental research. Further development of both in vitro and in silico model systems will undoubtedly provide new insights into somatic mutation and repair processes and their significance to tumorigenesis.

\section{Conclusions}

When inferring selection on single nucleotide variants it is standard practice to make comparisons between the observed variant frequencies and the frequencies expected, according to a model of single nucleotide mutation rates. We have developed models of DSB mutation rates that can be used to generate expected SV breakpoint frequencies and illuminate regions with significant deviations from these expectations. This approach provides statistically rigorous protocols to prioritize novel loci putatively under selection in tumors, generating testable hypotheses for further experimental studies.

\section{Methods}

\section{Derivation of DSB data in the K562 and MCF7 cell lines}

DSB profiles were generated with an adapted version of the Breaks labeling in situ and sequencing protocol [25], in which DSB ends are labeled with a dsDNA BLISS adapter in cell suspensions of one million cells. Afterwards, the published protocol is followed with only minor modifications. Labeled DSBs are selectively amplified using T7-driven linear amplification, after which sequencing libraries are generated and sequenced with single-end $1 \times 75$ v2 chemistry on an Illumina NextSeq 500. Raw sequencing reads were demultiplexed by Illumina's BaseSpace, after which FASTQ files were downloaded and processed as described in Yan et al. 2017
(SRA accession SRP150602). In brief, reads with the expected prefix of $8 \mathrm{nt}$ UMI and $8 \mathrm{nt}$ sample barcode sequence were filtered using SAMtools and scan for matches, allowing at most one mismatch per barcode. Trimmed reads were then aligned to GRCh37 using bwa mem, and reads with mapping scores below 30 were discarded. Next, PCR duplicates were identified by searching for proximal reads (within $30 \mathrm{bp}$ of the reference genome) with at most two mismatches in the UMI sequence, which were then grouped and collapsed into a single break location. Finally, we generated bed files with DSB locations and the number of unique UMIs indicating that location.

\section{Generating random forest models}

We downloaded ten tracks from ENCODE for multiple chromatin marks, replication timing, open chromatin, several DNA binding proteins, and nucleosome pull-downs from the UCSC genome browser [53]. We used G-quadruplex data generated by Chambers et al., (GSE63874). In their study, they make separate bedgraph files available with the G-quadruplex density for each strand. We used the sum of the plus and minus strands in our analysis. The list of bigwig files used for each cell line along with their sources and graphical labels is in Additional file 2: Table S1. We used the bigWigAverageOverBed tool from the kentUtils tool library to produce average signal per $50 \mathrm{~kb}$ in non-overlapping windows across hg19 for each track. We combined the results to a single matrix per cell line composed of 61,903 rows, one for each $50 \mathrm{~kb}$ bin, and 11 columns, one for each chromatin or genomic feature. These feature matrices are available as supplementary data [54] and scatter plots of each feature with the NHEK DSBCapture data are shown in Additional file 1: Figure S3.

For the extended model in Additional file 1: Figure S4, we downloaded an additional nine features from the UCSC genome browser [53], which were processed in the same way as the ten ENCODE features used in the primary feature matrix. We also downloaded hic files for NHEK, K562, and HMEC cells generated from Rao et al. (GSE63525). We used their custom toolbox, Juicer, to calculate eigenvectors per chromosome, and generated $50 \mathrm{~kb}$ resolution eigenvector profiles using the bedGraphToBigWig and bigWigAverageOverBed tools from kentUtils. The figure labels and sources for these data are in Additional file 2: Table S2, and the extended feature matrices are in supplementary data [54].

We generated DSB frequency scores from each of the four HTS DSB profiling datasets: two in NHEK cells, one for K562, unpublished, and one for MCF7, unpublished. As mentioned in the results, two replicates for each of two DSB HTS profiling methods, DSBCapture and BLESS, were available from Lensing et al. [22]. We 
took the average per $50 \mathrm{~kb}$ of the replicates to create an NHEK DSBCapture profile and an NHEK BLESS profile. We combined three replicates of MCF7 BLISS data (via a sum operation) to serve as our MCF7 DSB profile. A fourth MCF7 BLISS dataset is available, but we excluded it from our analysis because it had a distinctly lower correlation to the other three datasets $(0.90-0.92$ as opposed to 0.97-0.99). These scores are available as supplementary bedgraph files [54].

We used the randomForest package in $\mathrm{R}$ to generate random forest models with 500 trees and five OOB permutations per tree (options ntree $=500, \mathrm{nPerm}=5$ ). To calculate variable importance, we used the importance command within the randomForest package (https://cran.r-project.org/web/packages/randomForest/index.html), which calculates the average prediction error rate (MSE) for each datapoint $(50 \mathrm{~kb}$ bin) across all trees in the random forest. Then, for each feature variable, the values are randomly permuted and the MSE for each $50 \mathrm{~kb}$ bin is calculated again. The final variable importance score is the average difference in MSE before and after the permutation, normalized by the standard deviation of these differences. Because many features are inter-correlated, their importance measures were very similar. Therefore, in order to determine a consistent ranking of features' importance values, we generated ten random forest models per dataset and calculated the average and standard deviation of importance across the ten models.

Although random forest models are not susceptible to overfitting, to confirm that our models were not overfit to the DSB data, we also generated a random forest model for the NHEK DSBCapture dataset, holding out one third of the data as the test set and training the model on the remaining two thirds. This model showed 0.93 Pearson's correlation between the predictions and the observed data for the training set, similar to the model trained on the full dataset (Additional file 1: Figure S5).

\section{Determining tumor ESBs and their predicted DSB scores}

To determine SV DSB rates from TCGA data, we downloaded CNV data from TCGA [55], which came from Affymetrix SNP 6.0 arrays processed by the DNAcopy R-package (https://docs.gdc.cancer.gov/Data/PDF/Data _UG.pdf). DNAcopy generates a set of continuous segments, outputting regions with little or no copy number change, so we filtered these, defining segments with a $\mathrm{CN}$ ratio $>1$ as amplifications and ratios $<-1$ as deletions. The segments were lifted from hg38 to hg19 using UCSC's liftOver tool. For each CNV, we counted a single DSB to occur in a $50 \mathrm{~kb}$ bin if either or both ends of the segment overlapped the bin. The TCGA-BLOOD group includes the two blood cancer cohorts: acute myeloid leukemia (LAML) and lymphoid neoplasm diffuse large B cell lymphoma (DLBC), while the TCGA-SCCA group includes three squamous cell carcinomas: cervical squamous cell carcinoma and endocervical adenocarcinoma (CESC), head and neck squamous cell carcinoma (HNSC), and lung squamous cell carcinoma (LUSC). The BRCA group includes only the TCGA breast cancer cohort (BRCA), and the PANC group includes all 33 cancer types, shown in Additional file 1: Figure S7. Counts for various groups and CNV types are available as supplementary data [54].

We downloaded available WGS SV calls from the ICGC Data Portal (https://dcc.icgc.org/projects). As with the TCGA CNV, a single DSB was counted per $50 \mathrm{~kb}$ bin if either one or two ends of a SV overlapped the region. The ICGC pancancer group contains SVs from 17 cancer studies, shown in Additional file 1: Figure S6. The carcinoma group contains all available carcinoma cancer studies, excluding breast cancer: early-onset prostate cancer (EOPC-DE), liver cancer (LIRI-JP), pancreatic cancer (PACA-CA, PAEN-AU, PAEN-IT), prostate cancer (PRAD-CA, PRAD-UK), and skin adenocarcinoma (SKCA-BR). The ICGC blood group contains chronic lymphocytic leukemia (CLLE-ES) and malignant lymphoma (MALY-DE), and the breast group contains breast cancer studies (BRCA-EU and BRCA-FR). A table of DSB counts per $50 \mathrm{~kb}$ broken up by group and SV type is in supplementary data [54].

We determined enriched SV breakpoint regions (ESBs) per cohort or SV type grouping by ranking the $50 \mathrm{~kb}$ bins by the number of DSB, excluding regions with no DSB in the group, and using the number of DSB in the top $5 \%$ as the cutoff. All $50 \mathrm{~kb}$ regions with a DSB count greater than or equal to the cutoff were designated ESBs. We used a Wilcoxon ranked sum test ( $\mathrm{R}$ wilcox.test command) to test for a significant increase in the predicted DSB values for ESBs compared to all other regions, and we excluded regions in which no DSB were found in any cancer study since these are likely to be unmappable or blacklisted regions.

The correlation between TCGA and ICGC pancancer SV breakpoint counts was calculated using Spearman's rho and excluding $50 \mathrm{~kb}$ regions with no SV breakpoints in either the TCGA or ICGC datasets. The top 5\% ESBs were found for each dataset, with 2839 regions found in TCGA and 3072 in ICGC, and the significance of the overlap was calculated using a hypergeometric test $(\mathrm{R}$ command phyper with $q=177, m=2839, n=61,903$ 2839 , and $k=3072$ ).

\section{Calculating $d$-scores}

We used the $\mathrm{R}$ package fistdistrplus [56] to determine the distributions with the best fit to the DSB prediction values and the SV breakpoint frequencies. We used a likelihood maximization test (method = "mle") and the BIC (Bayesian Information Criterion) measure of 
goodness of fit to choose the best distribution. We tested a lognormal, log-logistic, gamma, normal, and an exponential distribution, and fitted the distributions to the bulk of the SV breakpoint or DSB prediction data. We excluded $50 \mathrm{~kb}$ regions with breakpoint frequencies greater than six times the interquartile range from the median in order to exclude extreme outliers. While we aimed to emphasize the fit of the tails of our data's distributions, including these outliers resulted in poorly fitting distributions to the bulk of the real data. Once we found the best of the three candidate model distributions, we assigned a $p$ value to each $50 \mathrm{~kb}$ bin from the fitted distribution (using the plnorm, pllogis, or pgamma functions in R) which represent the probability of seeing a given breakpoint frequency or DSB prediction or greater in the known distribution. The actual and fitted distributions and quantile-quantile plots are shown in Additional file 1: Figure S13 and S14.

Next, for each $50 \mathrm{~kb}$ bin, we calculated the difference in $\log p$ values between the predicted DSB and the actual SV breakpoints, called $d$-scores. Using the fistdistrplus R package again, we determined the best-fit distribution for the $d$-scores, choosing between a $t$-distribution, a normal, and a Cauchy distribution. Again, we used a maximum likelihood method and the BIC measurement and excluded extreme outliers. In all cases, a $t$-distribution with four degrees of freedom $(\mathrm{df}=4)$ was the best fit, so each $50 \mathrm{~kb}$ bin was assigned a $p$ value from this distribution according to its $d$-score. The histograms and quantile-quantile plots of the $d$-scores and fitted distributions are shown in Additional file 1: Figure S15.

\section{Calculating gene set and chromatin domain enrichments}

We used the $d$-score $p$ values to categorize regions into informative subsets, using the $\mathrm{R}$ command qt ( $p=0.01$, $\mathrm{df}=4$, lower.tail $=$ FALSE) to determine the $d$-score cutoffs. The cancHpredL class of regions have $d$-scores in the upper one percentile $(>3.75)$, and the cancLpredH have $d$-scores in the lower one percentile $(<-3.75)$. The cancHpredH class has $d$-scores in the 40th to 70th percentiles and SV breakpoint frequencies or DSB predictions with $p$ values less than 0.01 , so these regions have significantly ( $p$ value $<0.01$ ) high SV breakpoints or DSB predictions but insignificant $d$-scores ( $p$ value $<0.6)$. The cancHpredL2 class consists of regions with SV breakpoint $p$ values less than 0.01 , and DSB predictions less than 0.5 for the NHEK models and less than 0.001 for the MCF7 model.

We used a binomial test to measure the significance of overlaps between sets when comparing results from the MCF7 model and the NHEK model applied to ICGC breast cancer data and MCF7 cell line features (R command binom.test).
We used the $\mathrm{R}$ package regioneR [57] to compute the overlap significance between each set of regions and various genome and chromatin annotation files. A list of annotation sets and their original sources are in Additional file 2: Table S3. We matched Cancer5000 genes and Cosmic gene lists to RefSeq gene names in order to get their genome coordinates, so the cancer gene lists are RefSeq gene subsets. The super enhancer set (SEA) came from A549 cells, derived from a lung carcinoma [47]. Common fragile sites (CFS) were collected from NCBI's gene archive by searching for "common fragile site" or "fragile site" within human genes. Many fragile sites are annotated by chromosome band but do not have exact coordinates; we filtered these out because they are low resolution. The chromHMM [48] annotation came from the UCSC genome browser. We tested enrichment of the NHEK states with the NHEK model $d$-score classes and the HMEC track, from primary mammary epithelial cells, with the MCF7 model's $d$-score classes. The regioneR package performs random circular permutation of regions of interest and then computes the number of overlaps between the permutated set and a second set of regions. The $p$ value represents how often, over the course of the permutations, the two sets overlap to the same extent that they do without any permutation. We used 1000 iterations to achieve a maximum $p$ value of 0.001 .

\section{Additional files}

\begin{abstract}
Additional file 1: Figure S1. Correlations between DSB profiles from three DSB HTS experiments from three different cell types at $50 \mathrm{~kb}$ resolution. Figure S2. Chromatin and genomic features are intercorrelated. Figure S3. Correlations between individual genomic features and DSB frequency from NHEK DSBCapture data. Figure S4. Hi-C eigenvector data improves the random forest model for DSB frequency. Figure S5. Predicted versus observed values for one third of the NHEK DSBCapture data where the random forest model was trained on the other two thirds of the data. Figure S6. ICGC SV types and cohorts. Figure S7. TCGA CNV data across cancer types. Figure S8. Correlation between predicted DSB values and observed SV breaks. Figure S9. Correspondence between SV and DSB shown for NHEK DSBCapture data and ICGC pancancer SV calls. Figure S10. TCGA CNV breakpoint enriched regions have elevated DSB frequencies in blood cancers. Figure S11. Inference of positively and negatively selected regions in ICGC breast cancers. Figure S12. Chromatin state enrichments for $d$-score classes. Figure S13. Finding the best-fit distribution for SV breakpoint frequencies. Figure S14. Finding the best-fit distribution for predicted DSB values. Figure S15. Finding the best-fit distribution for $d$-score frequencies. (DOCX $12760 \mathrm{~kb})$
\end{abstract}

Additional file 2: Tables S1. bigWig files from http://hgdownload.soe.ucsc .edu/gbdb/hg19/bbi/ used to create feature sets. Table S2. Extended NHEK Feature Set. Table S3. Sources of annotation files and their names. (XLSX $62 \mathrm{~kb}$ )

\section{Acknowledgements}

We are indebted to the ICGC and TCGA projects for the timely public release of tumor genome sequencing data and SV calls.

\section{Funding}

This study was funded by core funding of the UK Medical Research Council (MRC) to the MRC Human Genetics Unit to C.S.; by grants from the Karolinska Institutet, the Ragnar Söderberg Foundation, the Swedish Foundation for Strategic Research (N.C.: BD15-0095), and the Strategic 
Research Programme in Cancer (StratCan) at Karolinska Institutet to N.C.; and by a Rubicon fellowship from the Netherlands Organisation for Scientific Research (NWO) to B.B.

\section{Availability of data and materials}

All analysis was done using GRCh37 as the reference genome. The raw BLISS sequencing data is available on SRA with accession SRP150602 (https:// www.ncbi.nlm.nih.gov/sra/SRP150602) [58]. All scripts and commands used to do this analysis are available on github [59] (https://github.com/ TracyBallinger/dsb_model). In addition, we have made iPython notebooks for the figures used in this manuscript to ease reproducibility and allow further exploration of the data, also available on github. The scripts, iPython notebooks, and all additional data files generated and used in this analysis can also be downloaded from zenodo and licensed under the MIT open source license [54] (https://zenodo.org/record/2537101\#.XDdwg8_7R24).

\section{Authors' contributions}

BB and RM generated the BLISS DSB profiles. SG developed the BLISS alignment pipeline and generated .bed files of DSB profiles. TB performed all subsequent data analysis and produced figures. TB and CS wrote the manuscript. NC and CS supervised the project. TB, BB, NC, and CS edited the final manuscript. All authors read and approved the final manuscript.

\section{Ethics approval and consent to participate}

Approval for access and use of ICGC variant data was obtained from the ICGC Data Access Compliance Office. Use of TCGA CNV does not require ethics approval.

\section{Consent for publication}

Not applicable

\section{Competing interests}

The authors declare that they have no competing interests.

\section{Publisher's Note}

Springer Nature remains neutral with regard to jurisdictional claims in published maps and institutional affiliations.

\section{Author details}

${ }^{1}$ MRC Human Genetics Unit, MRC Institute of Genetics and Molecular Medicine, University of Edinburgh, Crewe Road, Edinburgh EH4 2XU, UK. ${ }^{2}$ Science for Life Laboratory, Department of Medical Biochemistry and Biophysics, Karolinska Institutet, Stockholm, Sweden.

\section{Received: 10 October 2018 Accepted: 17 January 2019}

\section{Published online: 08 February 2019}

\section{References}

1. Ciriello G, Miller ML, Aksoy BA, Senbabaoglu Y, Schultz N, Sander C. Emerging landscape of oncogenic signatures across human cancers. Nat Genet. 2013;45(10):1127-33.

2. Patch A-M, Christie EL, Etemadmoghadam D, Garsed DW, George J, Fereday $\mathrm{S}$, et al. Whole-genome characterization of chemoresistant ovarian cancer. Nature. 2015:521(7553):489-94.

3. Scarpa A, Chang DK, Nones K, Corbo V, Patch A-M, Bailey P, et al. Wholegenome landscape of pancreatic neuroendocrine tumors. Nature. 2017; 543(7643):65-71.

4. Alaei-Mahabadi B, Bhadury J, Karlsson JW, Nilsson JA, Larsson E. Global analysis of somatic structural genomic alterations and their impact on gene expression in diverse human cancers. Proc Natl Acad Sci U S A. 2016; 113(48):13768-73.

5. Li Y, Roberts N, Weischenfeldt J, Wala JA, Shapira O, Schumacher S, et al. Patterns of structural variation in human cancer. bioRxiv 181339. https://doi. org/10.1101/181339.

6. Sudmant PH, Rausch T, Gardner EJ, Handsaker RE, Abyzov A, Huddleston J, et al. An integrated map of structural variation in 2,504 human genomes. Nature. 2015:526(7571):75-81.

7. Weischenfeldt J, Dubash T, Drainas AP, Mardin BR, Chen Y, Stütz AM, et al. Pan-cancer analysis of somatic copy-number alterations implicates IRS4 and IGF2 in enhancer hijacking. Nat Genet. 2017;49(1):65-74.
8. Glodzik D, Morganella S, Davies H, Simpson PT, Li Y, Zou X, et al. A somatic-mutational process recurrently duplicates germline susceptibility loci and tissue-specific super-enhancers in breast cancers. Nat Genet. 2017:49(3):341-8.

9. Morganella S, Alexandrov LB, Glodzik D, Zou X, Davies H, Staaf J, et al. The topography of mutational processes in breast cancer genomes. Nat Commun. 2016;7:11383.

10. Schuster-Böckler B, Lehner B. Chromatin organization is a major influence on regional mutation rates in human cancer cells. Nature. 2012;488(7412):504-7.

11. Ding L, Getz G, Wheeler DA, Mardis ER, McLellan MD, Cibulskis K, et al. Somatic mutations affect key pathways in lung adenocarcinoma. Nature. 2008;455(7216):1069-75.

12. Jackson SP, Bartek J. The DNA-damage response in human biology and disease. Nature. 2009;461(7267):1071-8.

13. Biehs $R$, Steinlage M, Barton $O$, Juhász S, Künzel J, Spies J, et al. DNA double strand break resection occurs during non-homologous end joining in G1 but is distinct from resection during homologous recombination. Mol Cell. 2017;65(4):671-684.e5.

14. Nussenzweig A, Nussenzweig MC. A backup DNA repair pathway moves to the forefront. Cell. 2007;131(2):223-5.

15. Clouaire T, Legube G. DNA double strand break repair pathway choice: a chromatin based decision? Nucl Austin Tex. 2015;6(2):107-13.

16. Glover TW, Berger C, Coyle J, Echo B. DNA polymerase alpha inhibition by aphidicolin induces gaps and breaks at common fragile sites in human chromosomes. Hum Genet. 1984;67(2):136-42

17. Canela A, Sridharan S, Sciascia N, Tubbs A, Meltzer P, Sleckman BP, et al. DNA breaks and end resection measured genome-wide by end sequencing. Mol Cell. 2016;63(5):898-911.

18. Crosetto N, Mitra A, Silva MJ, Bienko M, Dojer N, Wang Q, et al. Nucleotideresolution DNA double strand break mapping by next-generation sequencing. Nat Methods. 2013;10(4):361-5.

19. Frock RL, Hu J, Meyers RM, Ho Y-J, Kii E, Alt FW. Genome-wide detection of DNA double stranded breaks induced by engineered nucleases. Nat Biotechnol. 2015:33(2):179-86.

20. lacovoni JS, Caron P, Lassadi I, Nicolas E, Massip L, Trouche D, et al. Highresolution profiling of gammaH2AX around DNA double strand breaks in the mammalian genome. EMBO J. 2010;29(8):1446-57.

21. Kim D, Bae S, Park J, Kim E, Kim S, Yu HR, et al. Digenome-seq: genomewide profiling of CRISPR-Cas9 off-target effects in human cells. Nat Methods. 2015:12(3):237-43 1 p following 243.

22. Lensing SV, Marsico G, Hänsel-Hertsch R, Lam EY, Tannahill D, Balasubramanian S. DSBCapture: in situ capture and sequencing of DNA breaks. Nat Methods. 2016;13(10):855-7.

23. Slaymaker IM, Gao L, Zetsche B, Scott DA, Yan WX, Zhang F. Rationally engineered Cas9 nucleases with improved specificity. Science. 2016; 351(6268):84-8.

24. Wei P-C, Chang AN, Kao J, Du Z, Meyers RM, Alt FW, et al. Long neural genes harbor recurrent DNA break clusters in neural stem/progenitor cells. Cell. 2016;164(4):644-55.

25. Yan WX, Mirzazadeh R, Garnerone S, Scott D, Schneider MW, Kallas T, et al. BLISS is a versatile and quantitative method for genome-wide profiling of DNA double strand breaks. Nat Commun. 2017:8:15058.

26. De S, Michor F. DNA secondary structures and epigenetic determinants of cancer genome evolution. Nat Struct 38. Mol Biol. 2011;18(8):950-5.

27. Moore BL, Aitken S, Semple CA. Integrative modeling reveals the principles of multi-scale chromatin boundary formation in human nuclear organization. Genome Biol. 2015;16(1):1270.

28. Polak P, Karlic R, Koren A, Thurman R, Sandstrom R, Lawrence MS, et al. Cellof-origin chromatin organization shapes the mutational landscape of cancer. Nature. 2015;518(7539):360-4.

29. Whalen S, Truty RM, Pollard KS. Enhancer-promoter interactions are encoded by complex genomic signatures on looping chromatin. Nat Genet. 2016;48(5):488-96.

30. Consortium TEP. An integrated encyclopedia of DNA elements in the human genome. Nature. 2012;489(7414):57-74.

31. Chambers VS, Marsico G, Boutell JM, Di Antonio M, Smith GP Balasubramanian S. High-throughput sequencing of DNA G-quadruplex structures in the human genome. Nat Biotechnol. 2015:33(8):877-81.

32. Rao SSP, Huntley MH, Durand NC, Stamenova EK, Bochkov ID, Robinson JT, et al. A 3D map of the human genome at kilobase resolution reveals principles of chromatin looping. Cell. 2014;159(7):1665-80. 
33. Drier Y, Lawrence MS, Carter SL, Stewart C, Gabriel SB, Lander ES, et al. Somatic rearrangements across cancer reveal classes of samples with distinct patterns of DNA breakage and rearrangement-induced hypermutability. Genome Res. 2013;23(2):228-35.

34. Mourad R, Ginalski K, Legube G, Cuvier O. Predicting double strand DNA breaks using epigenome marks or DNA at kilobase resolution. Genome Biol. 2018;19(1):34

35. Canela A, Maman Y, Jung S, Wong N, Callen E, Day A, et al. Genome organization drives chromosome fragility. Cell. 2017;170(3):507-521.e18.

36. Kaiser VB and Semple CA. When TADs go bad: chromatin structure and nuclear organisation in human disease [version 1; referees: 2 approved]. F1000Research2017, 6(F1000 Faculty Rev):314. https://doi.org/10.12688/ f1000research.10792.1.

37. Altemose N, Miga KH, Maggioni M, Willard HF. Genomic characterization of large heterochromatic gaps in the human genome assembly. PLoS Comput Biol. 2014;10(5):e1003628

38. Glover TW, Wilson TE, Arlt MF. Fragile sites in cancer: more than meets the eye. Nat Rev Cancer. 2017;17(8):489-501.

39. Quint KD, Genders RE, de Koning MN, Borgogna C, Gariglio M, Bavinck JNB, et al. Human Beta-papillomavirus infection and keratinocyte carcinomas. J Pathol. 2015;235(2):342-54

40. Zhang J, Baran J, Cros A, Guberman JM, Haider S, Hsu J, et al. International Cancer Genome Consortium Data Portal-a one-stop shop for cancer genomics data. Database. 2011;2011(0):bar026.

41. Weckselblatt $B$, Rudd MK. Human structural variation: mechanisms of chromosome rearrangements. Trends Genet. 2015;31(10):587-99.

42. Viguera E, Canceill D, Ehrlich SD. Replication slippage involves DNA polymerase pausing and dissociation. EMBO J. 2001;20(10):2587-95.

43. Fungtammasan A, Walsh E, Chiaromonte F, Eckert KA, Makova KD. A genome-wide analysis of common fragile sites: what features determine chromosomal instability in the human genome? Genome Res. 2012;22(6): 993-1005.

44. Irony-Tur Sinai M, Kerem B. DNA replication stress drives fragile site instability. Mutat Res. 2018;808:56-61.

45. Lawrence MS, Stojanov P, Mermel CH, Robinson JT, Garraway LA, Golub TR, et al. Discovery and saturation analysis of cancer genes across 21 tumor types. Nature. 2014;505(7484):495-501.

46. Forbes SA, Beare D, Boutselakis H, Bamford S, Bindal N, Tate J, et al. COSMIC: somatic cancer genetics at high-resolution. Nucleic Acids Res. 2017;45(D1):D777-83.

47. Wei Y, Zhang S, Shang S, Zhang B, Li S, Wang X, et al. SEA: a superenhancer archive. Nucleic Acids Res. 2016;44(D1):D172-9.

48. Ernst J, Kellis M. ChromHMM: automating chromatin-state discovery and characterization. Nat Methods. 2012;9(3):215-6.

49. Mrasek K, Schoder C, Teichmann A-C, Behr K, Franze B, Wilhelm K, et al. Global screening and extended nomenclature for 230 aphidicolininducible fragile sites, including 61 yet unreported ones. Int J Oncol. 2010;36(4):929-40

50. Sima J, Gilbert DM. Complex correlations: replication timing and mutational landscapes during cancer and genome evolution. Curr Opin Genet Dev. 2014;25:93-100.

51. Roerink SF, Sasaki N, Lee-Six H, Young MD, Alexandrov LB, Behjati S, et al. Intra-tumor diversification in colorectal cancer at the single-cell level. Nature. 2018;556(7702):457-62.

52. Macheret M, Halazonetis TD. Intragenic origins due to short G1 phases underlie oncogene-induced DNA replication stress. Nature. 2018; 555(7694):112-6.

53. Kent WJ, Sugnet CW, Furey TS, Roskin KM, Pringle TH, Zahler AM, et al. The human genome browser at UCSC. Genome Res. 2002;12(6):996-1006.

54. Ballinger T, Bouwman B, Mirzazadeh R, Garnerone S, Crosetto N, Semple C. Data sets for modeling double strand break susceptibility and interrogating structural variation in cancer. zenodo.org. https://zenodo.org/record/ 2537101\#.XDd0y8_7R24. Accessed 10 Jan 2019.

55. Grossman RL, Heath AP, Ferretti V, Varmus HE, Lowy DR, Kibbe WA, et al. Toward a shared vision for cancer genomic data. N Engl J Med. 2016; 375(12):1109-12.

56. Delignette-Muller ML, Dutang C. fitdistrplus. An R Package for Fitting Distributions. Journal of Statistical Software. 2015;64(4):1-34. http://www. jstatsoft.org/v64/i04/.

57. Gel B, Díez-Villanueva A, Serra E, Buschbeck M, 2015. regioneR: an R/ Bioconductor package for the association analysis of genomic regions based on permutation tests. academic.oup.com.
58. Ballinger T, Bouwman B, Mirzazadeh R, Garnerone S, Crosetto N, Semple C. BLISS sequencing of MCF7 and K562 cells. NCBI SRA https://www.ncbi.nlm. nih.gov/sra/SRP150602. Accessed 10 Jan 2019.

59. Ballinger T, Bouwman B, Mirzazadeh R, Garnerone S, Crosetto N, Semple C. dsb_model. GitHub. https://github.com/TracyBallinger/dsb_model (2018).
Ready to submit your research? Choose BMC and benefit from:

- fast, convenient online submission

- thorough peer review by experienced researchers in your field

- rapid publication on acceptance

- support for research data, including large and complex data types

- gold Open Access which fosters wider collaboration and increased citations

- maximum visibility for your research: over $100 \mathrm{M}$ website views per year

At BMC, research is always in progress.

Learn more biomedcentral.com/submissions 